\title{
Article \\ Optimization of Pure-Component LNG Cascade Processes with Heat Integration
}

\author{
Oddmar Eiksund ${ }^{1, *}$, Eivind Brodal ${ }^{2}$ and Steven Jackson ${ }^{2}$ \\ 1 Department of Engineering and Safety, UiT The Arctic University of Norway, 9037 Tromsø, Norway \\ 2 Department of Engineering and Safety IVT, UiT The Arctic University of Norway, 9037 Tromsø, Norway; \\ eivind.brodal@uit.no (E.B.); steve.jackson@uit.no (S.J.) \\ * Correspondence: oddmar.eiksund@uit.no; Tel.: +47-77-66-03-38
}

Received: 23 November 2017; Accepted: 10 January 2018; Published: 15 January 2018

\begin{abstract}
Liquefaction of natural gas is an energy-intensive process in which the energy efficiency depends on the number of compressors stages and the heat integration scheme. The aim of the study is to systematically evaluate process performance of pure component cascade processes, present optimized designs for all relevant numbers of compression stages and compare energy consumption between processes with differing levels of complexity. An original method for the evaluation of process performance is developed that utilizes as little human interaction as possible, making it suitable for optimization. This study shows that a pure-component cascade process using the three refrigerants R290, R1150 and R50 must have at least 11 stages to equal the energy efficiency of the best mixed refrigerant process. An optimized configuration for an 11-stage process scheme operating at $20^{\circ} \mathrm{C}$ ambient temperature is described in detail.
\end{abstract}

Keywords: LNG; cascade; optimization; genetic algorithm; heat integration; compressor stages

\section{Introduction}

Natural gas (NG) power plants have high efficiencies and correspondingly low $\mathrm{CO}_{2}$ emissions compared to other fossil fuels. Due to this, and strong growth in gas supplies, liquefied natural gas (LNG) is widely accepted as a clean energy alternative to other fossil fuels where NG is not available through pipelines. LNG liquefaction is, however, an energy-intensive process and has correspondingly high $\mathrm{CO}_{2}$ emissions. An important way of reducing $\mathrm{CO}_{2}$ emissions from energy-intensive industries is to improve energy efficiency.

The first commercial NG liquefaction plant was built in 1964 in Algeria and was based on the pure-component cascade liquefaction process. Up to the 1990s, only two other LNG processes were used for LNG production: the single mixed-refrigerant (SMR) and propane precooled mixed-refrigerant (C3MR) processes [1]. Since the 1990s, development has focused on processes using mixed refrigerants, which can achieve higher efficiency than pure-component refrigerant processes (given the same number of stages). In 2006, the LNG plant at Melkøya in Northern Norway started production based on a mixed fluid cascade (MFC) process.

The Melkøya LNG plant is often claimed to be the most energy-efficient LNG plant in the world [2,3]. However, the energy efficiency of both pure-component and mixed refrigerant LNG processes varies fundamentally with the number of stages used. Pure-component processes can also offer other advantages such as efficient operation in off-design conditions [4] and can still be a competitive design in certain circumstances as illustrated by the opening of the Darwin pure-component cascade LNG plant in Australia (2006).

Optimization of LNG processes is complex due to the large number of possible design options. Process equipment such as compressors, separators, valves, expanders and heat exchangers are linked 
to each other by mass and heat balances and process parameters like temperature and pressure. Finding the most energy-efficient process with respect to equipment configuration therefore depends on a large search space. The problem is also typically nonlinear since adjusting operating conditions for one component automatically will change the operating conditions for the others. The search space becomes even larger as the number of pressure levels is increased.

Optimization of LNG processes has been studied for decades. In 1974, Barnés et al. investigated a few promising pure component cascade processes to identify optimum systems [5]. Heuristic rules have often been used to reduce the huge search space in optimization work of LNG processes. In 1980, Cheng et al. published a procedure for synthesizing refrigeration features [5] where they introduced a heuristic method to determine intermediate stages needed, based on an average compression work model. However, the increased computational power developed in recent decades allows for a much deeper analysis approach. Many different LNG plants and refrigerant compositions have been explored, optimized and compared [1,6]. For example, Wang et al. presented in 2005 a study describing a method to optimize the large number of design options in refrigeration and gas separation processes, using a genetic algorithm (GA) [7]. Stochastic methods like the GA have been widely used in optimization problems to avoid being trapped in local optima.

A literature review of the use of optimization in LNG process design and operation was recently published in 2014 by Austbø et al. [8] and includes a short summary of each of the 186 published works identified. Of the 186 published works, only seven consider pure-component cascade processes. Surprisingly, a generic design for pure-component cascade processes that operate at multiple pressure levels and with optimal heat integration has not been explored extensively in published papers. However, special cases with pure-component cascade processes like the Technip/Air Liquide Cascade (1964), Phillips Cascade (1969) and the Phillips' Optimized Cascade (1999) have been explored [6,9]. Kamalinezhad et al. [4] published a study in 2014 of a nine-stage (3-3-3) pure component process, comparing seven different scenarios for each refrigerant cycle. They developed a process software (LNG-Pro) for selecting the optimal synthesis of refrigeration systems with respect to minimizing the OPEX and CAPEX. Eaton et al. conclude that large LNG trains are both feasible and cost-effective when they studied the ConocoPhillips Optimized Cascade process [10].

The contribution of this study to the existing body of research into LNG process optimization is, therefore, to present a systematic study of a generic pure-component cascade liquefaction process where the process design (number of compressor stages and number and configuration of heat exchangers) is not constrained. In this work, pure refrigerant cascade processes with R290 (propane), R1150 (ethylene) and R50 (methane) have been studied in detail. Process performance and optimal designs are presented for all relevant numbers of pressure stages and energy consumption is compared for processes of varying complexity.

\section{Method}

A method to evaluate the most energy-efficient cascade design for a process with a fixed number of compressor stages is developed below. Efficiency is evaluated based on the total energy consumption per ton refrigerated natural gas $w_{\mathrm{NG}}(\mathrm{kWh} / \mathrm{ton})$, and the process design is optimized with respect to the number and configuration of the heat exchangers and the configuration of compressor stages. Only steady-state simulations are considered.

\subsection{Process Description}

The cascade processes considered here are based on R290, R1150 and R50. This represents the most common selection of pure-component refrigerants used in industrial liquefaction processes as typified by the proprietary Phillips Optimized Cascade process, which is describe (for example) by Lim et al. [1]. R290 is used in the first refrigeration circuit cooling the NG, and R50 is used in the last refrigeration circuit cooling the NG to $-155^{\circ} \mathrm{C}$. The total number of compressor stages in the cascade process $(N)$ is a sum of the individual compressor stages in each refrigerant cycle, 
i.e., $N=n_{R 290}+n_{R 1150}+n_{R 50}$ compressor stages. The configuration of compressor stages is important to overall process performance and the different designs are named $n_{R 290}-n_{R 1150}-n_{R 50}$-Cascade. Figure 1 illustrates a possible configuration of the simplest cascade process considered here (1-1-1-Cascade), using only $N=3$ compressor stages. Figure 2 shows the 1-2-1-Cascade process (only the ethylene circuit is shown in detail), where the single-stage R1150 in 1-1-1-Cascade has been replaced with a two stage compression process where R1150 is evaporated in two separate heat exchangers. This process is one of three configurations that have $N=4$ compressor stages. There are also many possible configurations of heat exchangers used in the cooling and condensing of the refrigerant, as illustrated by the dashed lines in Figure 2. A pressure $(p)$-enthalpy $(h)$ diagram for the R1150-circuits is shown in Figure 3.

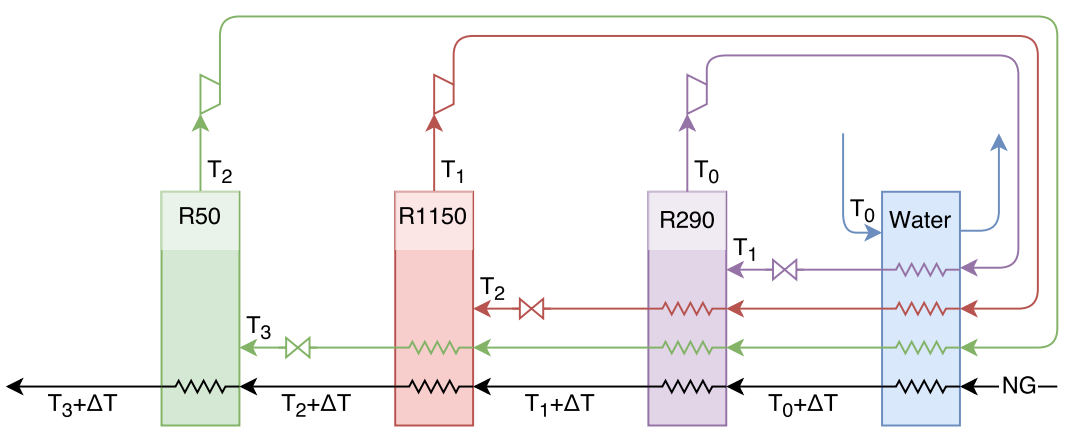

Figure 1. 1-1-1-Cascade.

Heat exchangers are modeled with pre-saturators, as explained by Kamalinezhad et al. [4] and Lim et al. [1] and illustrated in Figure 2 where the ethylene gas at $J$ is mixed with the liquid. In this configuration, the heat exchangers represent Coil-Wound Heat Exchangers (CWHE) where both natural gas and other refrigerant streams can be cooled against each other.

Evaporators, compressors, refrigerant cycles and temperature levels are organized similarly. For example, the process model includes $N+1$ different temperature levels $T_{n}$, i.e., $T_{0}, T_{1}, \ldots, T_{N}$, which are related to the natural gas temperature out of the seawater cooler and the $N$ evaporators as illustrated in Figure 1. The $T_{n}$ temperatures are organized in decreasing order, starting at ambient seawater temperature $T_{0}$. $T_{N}$ is the lowest temperature in the process, which is obtained in the last R50-evaporator.

In addition to the natural gas mass flow $\left(\dot{m}_{\mathrm{NG}}\right)$ and the seawater mass flow $\left(\dot{m}_{0}\right)$, there are $N$ different mass flows of refrigerants $\left(\dot{m}_{1}, \ldots, \dot{m}_{n}, \ldots, \dot{m}_{N}\right)$ through $N$ compressors. That is, refrigeration cycle number $n=3$ has evaporator temperature $T_{3}$, refrigerant mass flow $\dot{m}_{3}$ and compressor energy consumption $\dot{W}_{c, 3}$. Figure 3 shows a $p$ - $h$ diagram for the R1150 refrigeration cycle in the 1-2-1-Cascade process illustrated in Figure 2.

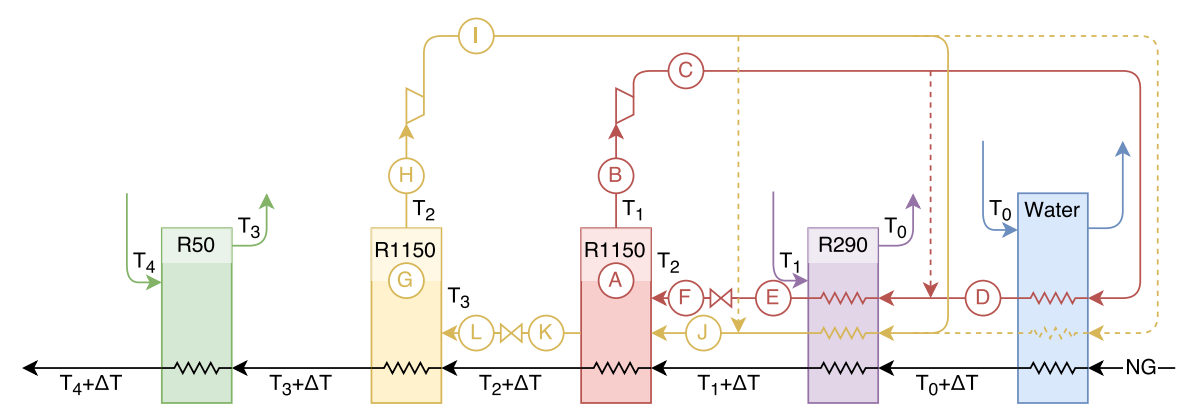

Figure 2. Optimized 1-2-1-Cascade, R1150 circuits. Optimized configuration (solid lines) and other possible configurations (dashed lines). 


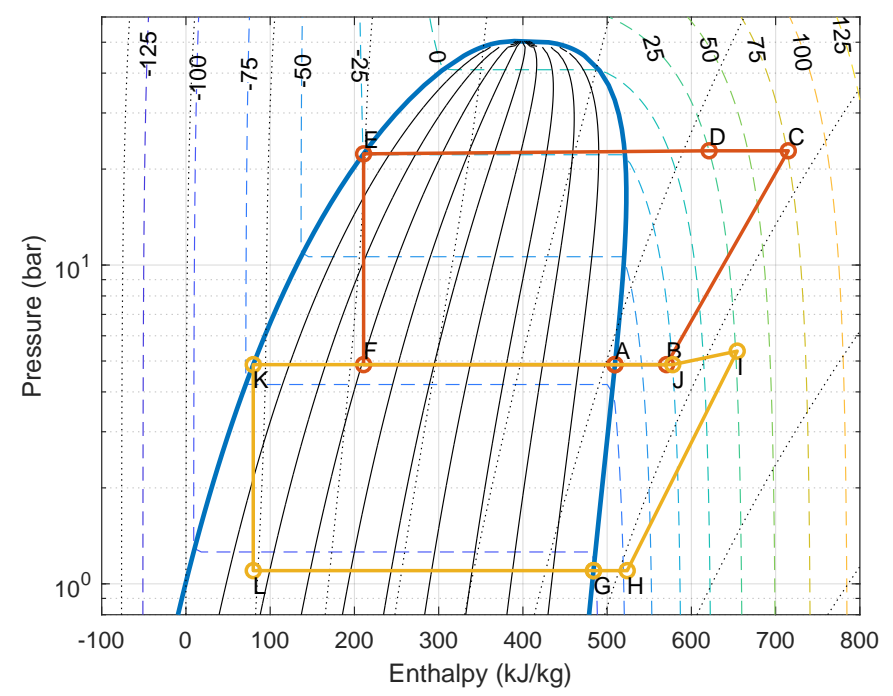

Figure 3. A $p$-h diagram for the R1150-circuits in the Optimized 1-2-1-Cascade.

It is assumed that all heat exchangers (condensers, evaporators and gas coolers) operate with a single common minimum temperature difference $\Delta T$ and that refrigerant vapor is superheated by the natural gas in the evaporators, as illustrated in Figures 1 and 2.

Modeling Parameters

- Work by equipment other than the refrigerant compressors, such as the seawater pumps, is neglected.

- Feed composition (in mol $\%$ ) is: $3.1 \%$ nitrogen, $87.7 \%$ methane, $5.4 \%$ ethane, $2.6 \%$ propane, $0.8 \%$ iButane and $0.4 \%$ nButane

- Feed gas pressure after the initial seawater cooler is 60 bar.

- All heat exchangers operate with a $\Delta T=5.0 \mathrm{~K}$ temperature difference.

- Streams flowing through exchangers without phase change have a $\Delta p=0.5$ bar pressure drop.

- CWHE evaporators are modeled with zero pressure drop outside the coils, i.e., neglecting pressure drops when superheating the boil off gas. Illustrated in Figures 2 and 3 where $p_{A}=p_{B}=p_{F}=p_{J}=p_{K}$.

- $\quad$ All compressors have isentropic efficiency $\eta_{i s}=0.85$.

- The lowest evaporation occurs at $T_{N}=-160^{\circ} \mathrm{C}$, satisfying the minimum pressure requirement for R50.

\subsection{Process Modeling}

Refrigerant and natural gas processes are calculated using the property library CoolProp [11]. This is done by letting CoolProp calculate specific enthalpies $h$ for the different thermodynamic states in the cascade process, e.g., by specifying temperature, pressure, gas quality or entropy.

The total energy consumption per ton refrigerated natural gas (feed gas rate) is calculated as the sum of all the $N$ compressor stages, since additional work by seawater pumps and other equipment is neglected:

$$
w_{\mathrm{NG}}=\frac{1}{\dot{m}_{\mathrm{NG}}} \sum_{n=1}^{N} \dot{W}_{c, n}=\frac{1}{\dot{m}_{\mathrm{NG}}} \sum_{n=1}^{N} \Delta h_{c, n} \dot{m}_{n}
$$

where $\dot{W}_{c, n}$ is the compressor power consumption in refrigerant circuit number $n$ and $\Delta h_{c, n}$ is the corresponding enthalpy change. Heat flow in the other components is calculated similarly. For example, 
heat flows in the second refrigerant cycle, linked to the compressor stage $n=2$, in the 1-2-1-Cascade shown in Figure 3 are:

$$
\begin{aligned}
& \dot{W}_{c, 2}=\dot{m}_{2} \cdot\left(h_{C}-h_{B}\right)=\dot{m}_{2} \cdot \Delta h_{c, 2} \\
& \dot{Q}_{e, 2}=\dot{m}_{2} \cdot\left(h_{B}-h_{F}\right)=\dot{m}_{2} \cdot \Delta h_{e, 2} \\
& \dot{Q}_{0,2}=\dot{m}_{2} \cdot\left(h_{C}-h_{D}\right)=-\dot{m}_{2} \cdot \Delta h_{0,2} \\
& \dot{Q}_{1,2}=\dot{m}_{2} \cdot\left(h_{D}-h_{E}\right)=-\dot{m}_{2} \cdot \Delta h_{1,2}
\end{aligned}
$$

where $\dot{Q}_{e, 2}$ is the transferred heat in Evaporator Number 2 (with boiling temperature $T_{2}$ ), $\dot{Q}_{0,2}$ is the transferred heat in the seawater exchanger and $\dot{Q}_{1,2}$ is the transferred heat in the exchanger related to the propane evaporator with boiling temperature $T_{1}$.

An energy balance for the evaporators, using a steady-state assumption, is used to find the mass flows. Evaporator number $n$ can be used to decide the mass flow through compressor number $n$ using the energy conservation equation:

$$
\begin{array}{r}
\dot{Q}_{e, n}-\sum_{i=n+1}^{N} \dot{Q}_{n, i}-\dot{Q}_{n, \mathrm{NG}}=0 \\
\dot{m}_{n} \cdot \Delta h_{e, n}+\sum_{i=n+1}^{N} \dot{m}_{i} \cdot \Delta h_{n, i}+\dot{m}_{\mathrm{NG}} \cdot \Delta h_{n, \mathrm{NG}}=0
\end{array}
$$

given that all mass flows $\dot{m}_{n+1}, \ldots, \dot{m}_{N}$ and $\dot{m}_{\mathrm{NG}}$ are known.

Table 1 shows the optimized configuration of the settings for a system with $N$ compressor stages. The $p$ - $h$ diagram in 1-2-1-Cascade with respect to heat exchanger, compressor and temperature Figure 3 illustrates this configuration for the R1150 refrigerant processes. The $n=2$ evaporator in the 1-2-1-Cascade is used to cool the refrigerant in the $n=3$ and $n=4$ circuits. The mass flows are found relative to the natural gas mass flow by solving the energy balance recursively bottom up. For the 1-2-1-Cascade, the mass flows are calculated by solving the matrix problem:

$$
\left[\begin{array}{cccccc}
\Delta h_{0}^{*} & \Delta h_{0,1} & \Delta h_{0,2} & 0 & \Delta h_{0,4} & \Delta h_{0, \mathrm{NG}}^{*} \\
0 & \Delta h_{e, 1} & \Delta h_{1,2} & \Delta h_{1,3} & \Delta h_{1,4} & \Delta h_{1, \mathrm{NG}} \\
0 & 0 & \Delta h_{e, 2} & \Delta h_{2,3} & \Delta h_{2,4} & \Delta h_{2, \mathrm{NG}} \\
0 & 0 & 0 & \Delta h_{e, 3} & \Delta h_{3,4} & \Delta h_{3, \mathrm{NG}} \\
0 & 0 & 0 & 0 & \Delta h_{e, 4} & \Delta h_{4, \mathrm{NG}} \\
0 & 0 & 0 & 0 & 0 & 1
\end{array}\right] \cdot\left[\begin{array}{c}
\dot{m}_{0} \\
\dot{m}_{1} \\
\dot{m}_{2} \\
\dot{m}_{3} \\
\dot{m}_{4} \\
\dot{m}_{\mathrm{NG}}
\end{array}\right]=\left[\begin{array}{l}
0 \\
0 \\
0 \\
0 \\
0 \\
1
\end{array}\right]
$$

where $\Delta h_{0}^{*}$ and $\Delta h_{0, \mathrm{NG}}^{*}$ depend respectively on the exiting temperature of the seawater and the feed temperature of the natural gas and are not specified in this article because of the modeling assumption that seawater cooling comes at no additional cost. That is, $\dot{m}_{0}$, corresponding to the first row in the matrix problem, is not computed.

Table 1. Optimized configuration of 1-2-1-Cascade. Heat Exchanger Number 2 is used as the condenser for Refrigerant Cycle 3 and as gas cooler for Cycle 4 as illustrated with boldface numbers below.

\begin{tabular}{ccccc}
\hline Circuit Number & Refrigerant & Evaporator Temperature $\left({ }^{\circ} \mathrm{C}\right)$ & Used Heat Ex. & $j_{n}$ \\
\hline 0 & Seawater & 20.0 & - & - \\
1 & R290 & -29.8 & 0 & 1 \\
2 & R1150 & -71.5 & 1,0 & 2 \\
3 & R1150 & -102.4 & $\mathbf{2}, 1$ & 2 \\
4 & R50 & -160.0 & $3, \mathbf{2}, 1,0$ & 4 \\
\hline
\end{tabular}


The missing $\Delta h_{0,3}$ in the first row is set to " 0 " since the third refrigerant circuit in the optimized 1-2-1-Cascade design does not use a seawater heat exchanger, as illustrated in Table 1 and Figure 3. There are many possible process plant designs for the 1-2-1-Cascade that are more energy intensive, and all but one (the condenser) of the off-diagonal heat exchangers in each column could in theory be zero, i.e., not used.

\subsection{Optimization}

Development of a method for finding the optimum cascade process given the number of compression stages $N$ is done in three steps:

1. Finding the best heat exchanger configuration given a compressor configuration $N=n_{R 290}-n_{R 1150}-n_{R 50}$ and all temperature levels.

2. Finding the best temperature levels given configuration $N=n_{R 290}-n_{R 1150}-n_{R 50}$.

3. Finding the the best compressor configuration given the number of compression stages $N$.

\subsubsection{Optimization of Heat Exchanging Equipment}

Even with all the temperature levels and compressor configuration defined, the cascade process is not uniquely determined, as illustrated in Figure 2 as dashed lines and the zeros in the matrix equation Equation (4). The initial approach used in the optimization model to decide how many heat exchangers should be included was to limit heat exchangers to cases where the temperature difference between two streams was greater than $\Delta T=5.0 \mathrm{~K}$. This approach was applied by Jackson et al.'s study of a 2-2-2-Cascade process in [12]. However, in some cases, the process plant can be less complex and have higher energy efficiency if some heat exchangers are excluded.

A bias towards the most energy-efficient process design is included using an additional constraint in the form of a recursive algorithm that compares the coefficient of performance (COP) of additional cooling cycles to that of the cycle they replace. The optimum COP is found by sequentially adding pre-coolers and searching for a maximum in the COP. Starting with the cycle with the highest evaporator temperature, this can be written as a recursive optimization problem:

$$
\begin{aligned}
\mathrm{COP}_{0} & =\infty \\
\mathrm{COP}_{1} & =\frac{\Delta h_{e, 1}}{\Delta h_{c, 1}} \\
\mathrm{COP}_{n} & =\max _{k \in\left\{1, \ldots, j_{n}\right\}} \frac{\Delta h_{e, n}}{\Delta h_{c, n}+\sum_{i=1}^{k} \frac{\Delta h_{(n-i), n}}{\operatorname{COP}_{n-i}}}
\end{aligned}
$$

where $\mathrm{COP}_{0}=\infty$ since power consumption from seawater pumps is neglected. The number $j_{n}$ is the maximum number of heat exchangers with usable energy for circuit number $n$. Note that $j_{n} \leq n$ and depends on the temperature levels, compressor efficiency $\eta$ and the pressure drop $\Delta p$. Table 1 shows the optimized result for the 1-2-1-Cascade.

\subsubsection{Optimization of Temperature Levels}

Our objective is to minimize the specific energy consumption by adjusting the temperature settings for the evaporators. Given the number of compressors $N$ and compressor configuration, $n_{R 290}+n_{R 1150}+n_{R 50}=N$, this can be regarded as a real-valued function of the temperatures:

$$
f_{\left(n_{R 290}, n_{R 1150,}, n_{R 50}\right)}:\left(T_{1}, \ldots, T_{N-1}\right) \mapsto w_{\mathrm{NG}}
$$

Both the ambient temperature $T_{0}$ and the final evaporator temperature $T_{N}$ are assumed to be fixed and excluded from the list of arguments. This function is constrained by the inequality $T_{0}>T_{1}>T_{2}>\cdots>T_{N}$. Figure 4 shows how a version of this function $f_{(2,2,2)}$ varies in a two-dimensional subspace and illustrates the complexity of finding the global minimum, due to 
other local minimums. MATLAB functions $g a$ and $f$ minsearch were tested to evaluate their ability to find the global minimum. The fminsearch approach often did not converge to the global minimum as it depends on a relatively good initial guess. The ga approach gave better results, but at the cost of longer runtime. A hybrid solver was therefore used for optimization, where ga was used to get close to the optimal configuration, and fminsearch was used to fine-tune the parameters, using the $g a$ solution as the initial guess. The hybrid approach versus computer runtime is illustrated in Figure 5.

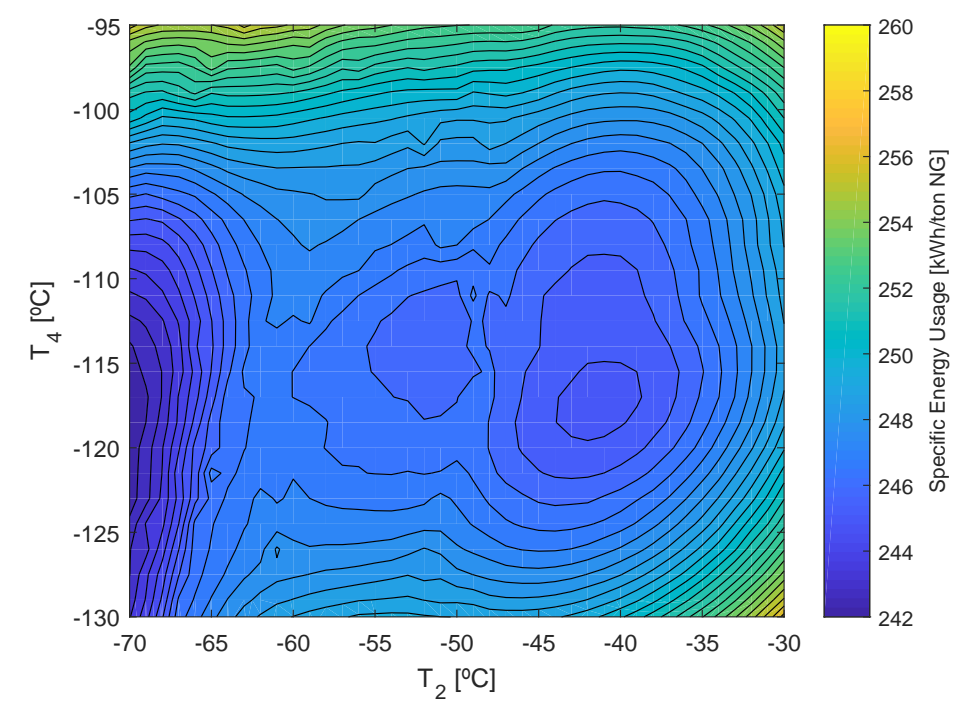

Figure 4. Illustration of locale minima in the specific energy consumption for partly optimized 2-2-2-Cascade processes where $T_{2}$ and $T_{4}$ are kept fixed in the optimization. The minimum pressure requirement is ignored in this illustrative example.

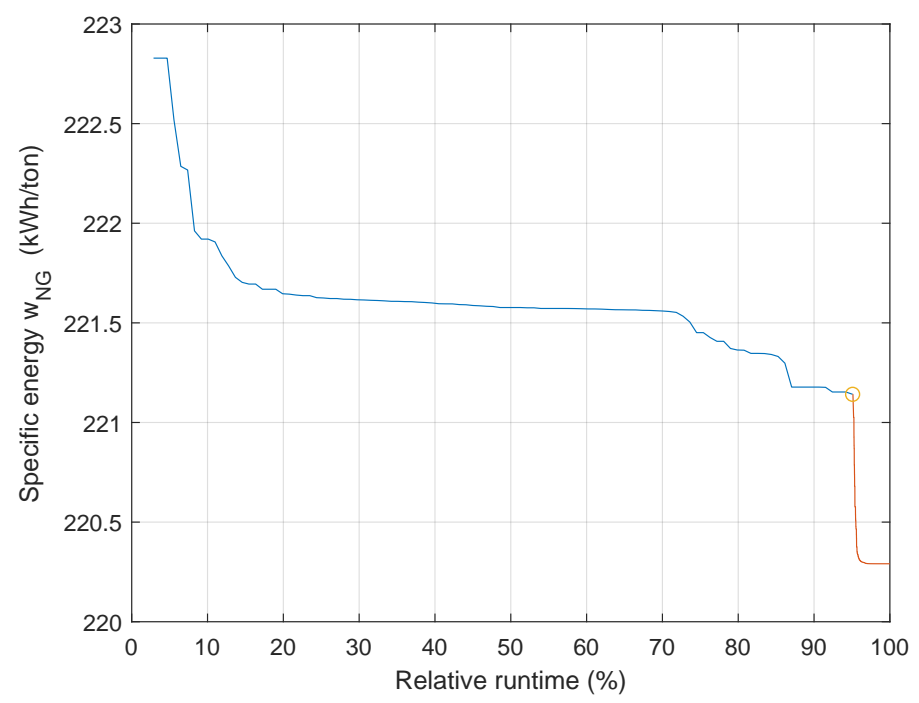

Figure 5. Computer runtime for the hybrid optimization approach with $g a$ (blue) and fminsearch (red).

In this work, evenly-distributed temperature levels in each refrigerant-cycle are used as the starting point for the optimization. In this configuration, and throughout the subsequent optimization process, a constraint is applied to the minimum operating pressure for each refrigerant cycle where $p_{\min } \geq 1.1$ bara. It turns out that this initial guess is relatively good as shown in Figure 6 . The gain by optimization is roughly $1.5 \%$ on average and as high as $4.0 \%$ in some cases. Since this initial guess 
approach is close to the optimal configuration, fminsearch is typically able to converge to the global minimum without using $g a$ first.

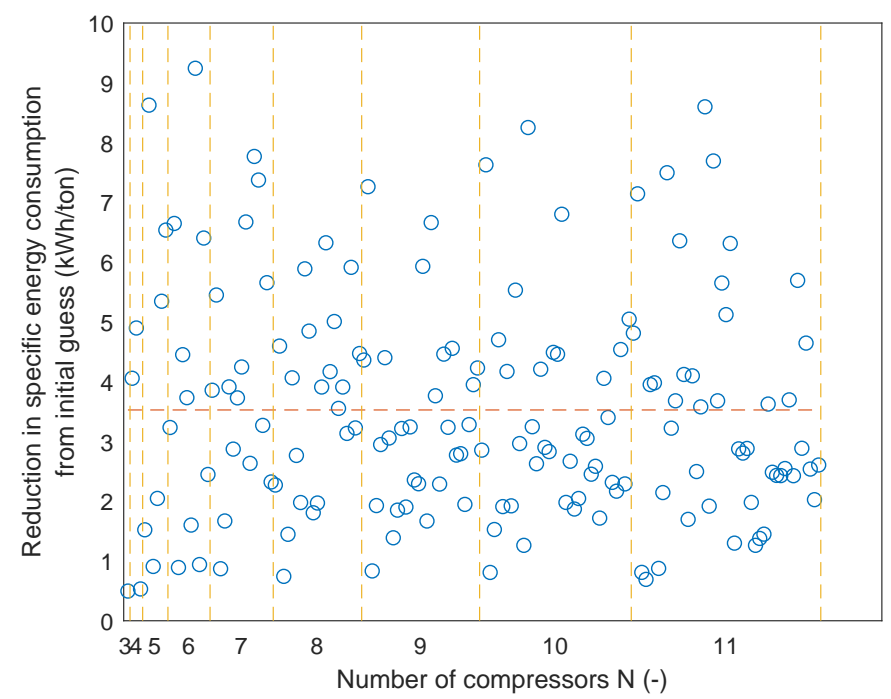

Figure 6. Reduction in specific energy usage when optimizing evenly distributed temperature levels for all modeled cases shown in Figure 7.

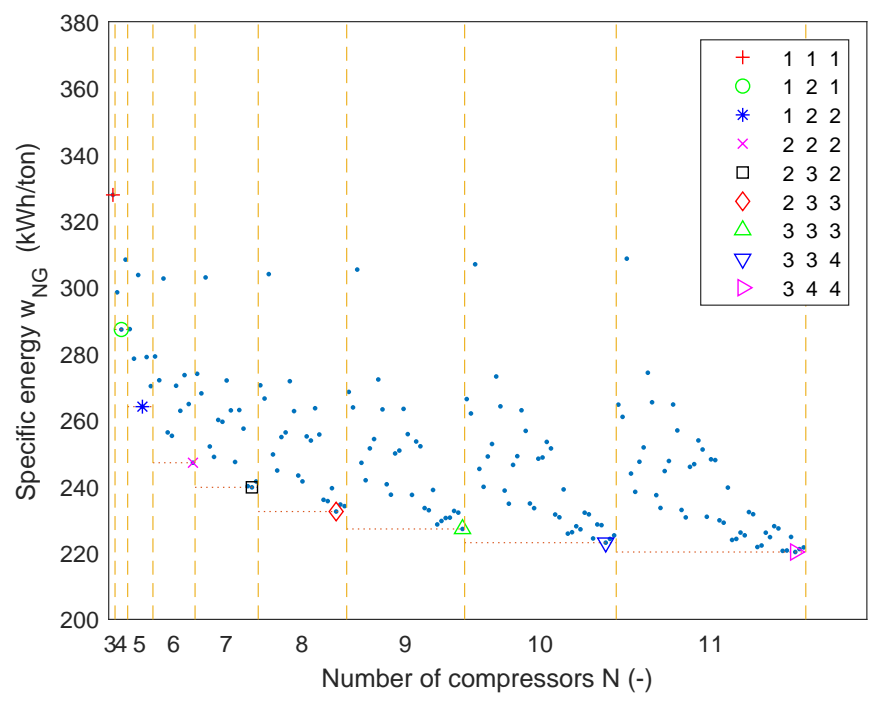

Figure 7. Optimized for all different $n_{R 290}-n_{R 1150}-n_{R 50}$-Cascade process configurations with $N=n_{R 290}+n_{R 1150}+n_{R 50}$ compressor stages.

\subsubsection{Optimization of Compressor Configuration}

Finding the most energy-efficient process design with $N$ compressor stages is done with an exhaustive search, i.e., modeling all possible configurations $n_{R 290}-n_{R 1150}-n_{R 50}$-Cascade with $n_{R 290}+n_{R 1150}+n_{R 50}=N$ and comparing their efficiencies. The total number of possibilities to examine is $\frac{(N-2)(N-1)}{2}$. All possibilities are examined to make the approach as general as possible.

\subsection{Model Verification}

To provide a high-level check of the process model developed in MATLAB, a sample of the results was tested against data generated using an independent process model. The independent model was developed in HYSYS with thermodynamic data generated using the standard HYSYS implementation 
of the Peng-Robinson equation of state. HYSYS models for the optimized 1-2-1-Cascade presented in Table 1 and three optimized 2-2-2-Cascade for ambient temperatures 10, 20 and $30^{\circ} \mathrm{C}$ showed a maximum $0.9 \%$ difference in the overall enthalpy change for the natural gas steam and a maximum $1.7 \%$ difference in $w_{\mathrm{NG}}$ compared to the MATLAB simulations.

\subsection{Comparative Study of Process Performance}

MFC processes are claimed to be the most energy-efficient processes currently used in an LNG plant [2,3], and are used a as references points in this article. MFC performance data are presented here for a fixed system with three mixed refrigerants based on a previous study concerned with the modeling of the optimized MFC LNG liquefaction process for a range of heat-sink temperature cases [12]. This article is highly relevant in the comparative study, since it uses the same modeling basis as the single component cascade processes presented here, i.e., the same gas composition, feed pressure, compressor efficiencies and ambient temperatures. A comparison of the result from these two studies is presented in Figure 8.

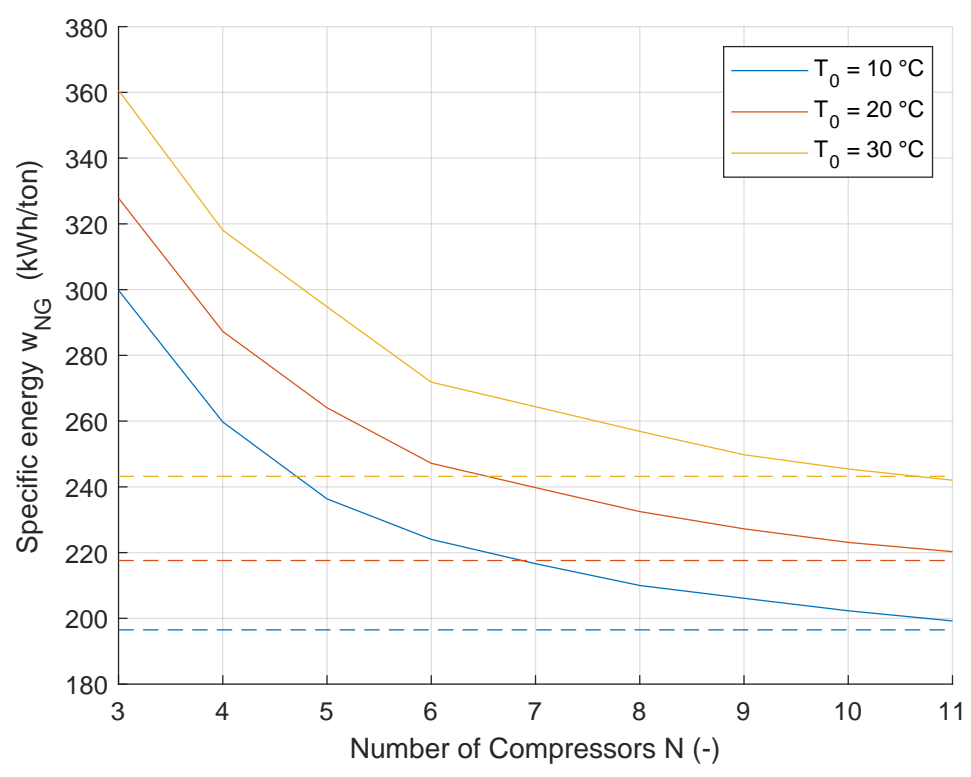

Figure 8. Variation of the specific energy consumption of optimized process with $N$ compressor stages and ambient seawater temperature $T_{0}$. Dashed lines are mixed fluid cascade (MFC) processes.

\section{Results}

Figure 7 presents energy consumption for different configurations with eleven or less compressor stages $(N \leq 11)$ optimized for $T_{0}=20.0^{\circ} \mathrm{C}$. The most efficient process for a given number of compression stages is marked in Figure 7 , and the optimized temperature levels $T_{0}, T_{1}, \ldots, T_{N}$ are shown in Figure 9. The 3-4-4-Cascade process is the most efficient process with a maximum of 11 compressor stages, and Table 2 describes the heat exchanger configuration for this particular optimized design. Optimized heat exchanger configuration and temperature settings for 1-2-1-Cascade and 3-4-4-Cascade are shown in Tables 1 and 2. Tables 3 and 4 show additional process parameters for the 3-4-4-Cascade.

Figure 8 shows the specific energy consumption for the most efficient processes with $N \leq 11$ for three different cases $T_{0}=10.0^{\circ} \mathrm{C}, T_{0}=20.0^{\circ} \mathrm{C}$ and $T_{0}=30.0^{\circ} \mathrm{C}$. Figure 10 shows the energy saved by adding an extra compressor stage, i.e., going from $N$ to $(N+1)$ stages. 
Table 2. Optimized configuration of the 3-4-4-Cascade.

\begin{tabular}{ccccc}
\hline Circuit No. & Refrig. & Evap. Temp. $\left({ }^{\circ}\right.$ C) & Used Heat Ex. & $j_{n}$ \\
\hline 0 & Seawater & 20.0 & - & - \\
1 & R290 & 1.7 & 0 & 1 \\
2 & R290 & -17.2 & 1 & 1 \\
3 & R290 & -40.2 & 2 & 3 \\
4 & R1150 & -61.5 & 3,2 & 4 \\
5 & R1150 & -73.0 & 4 & 1 \\
6 & R1150 & -87.1 & 5 & 1 \\
7 & R1150 & -102.4 & 6 & 3 \\
8 & R50 & -118.9 & $7,6,5$ & 3 \\
9 & R50 & -136.2 & 8,7 & 2 \\
10 & R50 & -148.1 & 9 & 1 \\
11 & R50 & -160.0 & 10 & 1 \\
\hline
\end{tabular}

Table 3. Heat exchanger power (kW/ton NG). Numbers in parentheses indicate the circuit number.

\begin{tabular}{cccc}
\hline Ex. No. & $\begin{array}{c}\text { Exchanger Duty } \\
\text { (kW/ton) }\end{array}$ & $\begin{array}{c}\text { Natural Gas Cooling } \\
\mathbf{( k W / t o n )}\end{array}$ & $\begin{array}{c}\text { Process Cooling } \\
\mathbf{( k W / t o n )}\end{array}$ \\
\hline 1 & 1.390 & 0.047 & $1.342(2)$ \\
2 & 1.226 & 0.052 & $1.056(3), 0.118(4)$ \\
3 & 0.935 & 0.074 & $0.862(4)$ \\
4 & 0.823 & 0.112 & $0.711(5)$ \\
5 & 0.661 & 0.145 & $0.489(6), 0.027(8)$ \\
6 & 0.444 & 0.082 & $0.335(7), 0.027(8)$ \\
7 & 0.299 & 0.062 & $0.218(8), 0.019(9)$ \\
8 & 0.217 & 0.059 & $0.158(9)$ \\
9 & 0.148 & 0.058 & $0.091(10)$ \\
10 & 0.080 & 0.038 & $0.043(11)$ \\
11 & 0.037 & 0.037 & - \\
\hline
\end{tabular}

Table 4. Process parameters for the optimized 3-4-4-Cascade. Mass Flow is defined as $\dot{m} / \dot{m}_{\mathrm{NG}}$.

\begin{tabular}{cccccc}
\hline Comp. No. & $\begin{array}{c}\text { Inlet Temp. } \\
\left({ }^{\circ} \mathbf{C}\right)\end{array}$ & $\begin{array}{c}\text { Inlet Pressure } \\
(\mathbf{b a r})\end{array}$ & $\begin{array}{c}\text { Pressure Ratio } \\
\mathbf{( - )}\end{array}$ & $\begin{array}{c}\text { Mass Flow } \\
(-)\end{array}$ & $\begin{array}{c}\text { Compressor Power } \\
(\mathbf{k W} / \text { ton NG) }\end{array}$ \\
\hline 1 & 20.0 & 4.99 & 2.01 & 4.04 & 47.13 \\
2 & 1.7 & 2.70 & 1.85 & 3.21 & 32.30 \\
3 & -17.2 & 1.10 & 2.46 & 2.31 & 33.40 \\
4 & -40.2 & 7.15 & 2.41 & 2.22 & 43.62 \\
5 & -61.5 & 4.58 & 1.56 & 1.58 & 13.88 \\
6 & -73.0 & 2.45 & 1.87 & 1.01 & 12.47 \\
7 & -87.1 & 1.10 & 2.23 & 0.65 & 9.99 \\
8 & -102.4 & 12.54 & 2.36 & 0.64 & 15.02 \\
9 & -118.9 & 5.44 & 2.39 & 0.35 & 8.12 \\
10 & -136.2 & 2.70 & 2.02 & 0.17 & 2.89 \\
11 & -148.1 & 1.14 & 2.36 & 0.08 & 1.48 \\
\hline
\end{tabular}




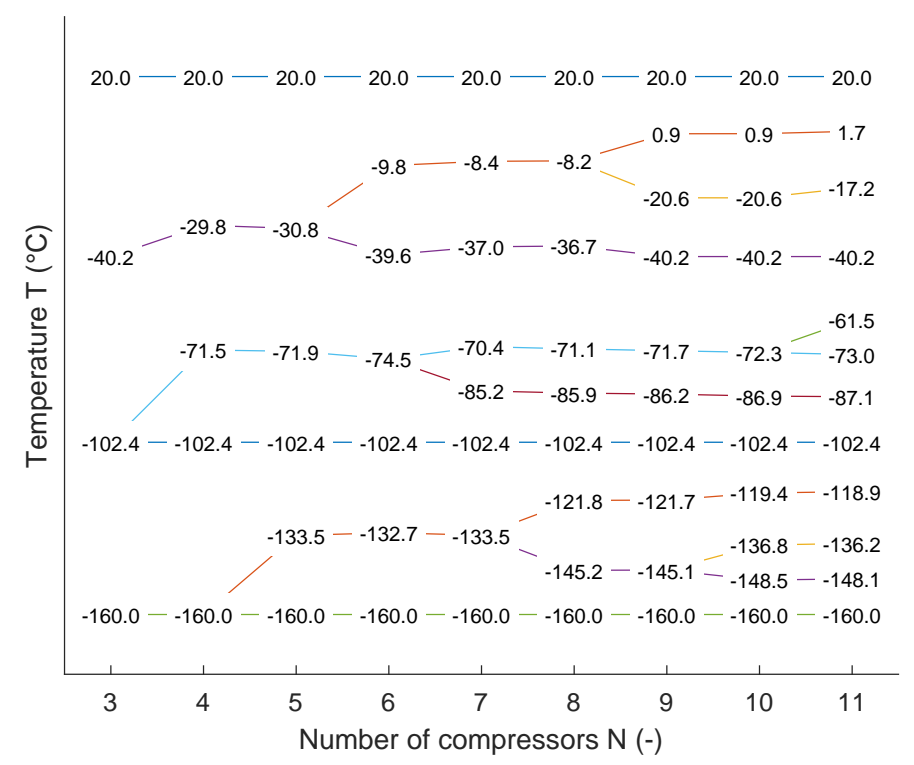

Figure 9. Temperature settings for the most energy-efficient cascade process with $N$ compressor stages at $T_{0}=20^{\circ} \mathrm{C}$.

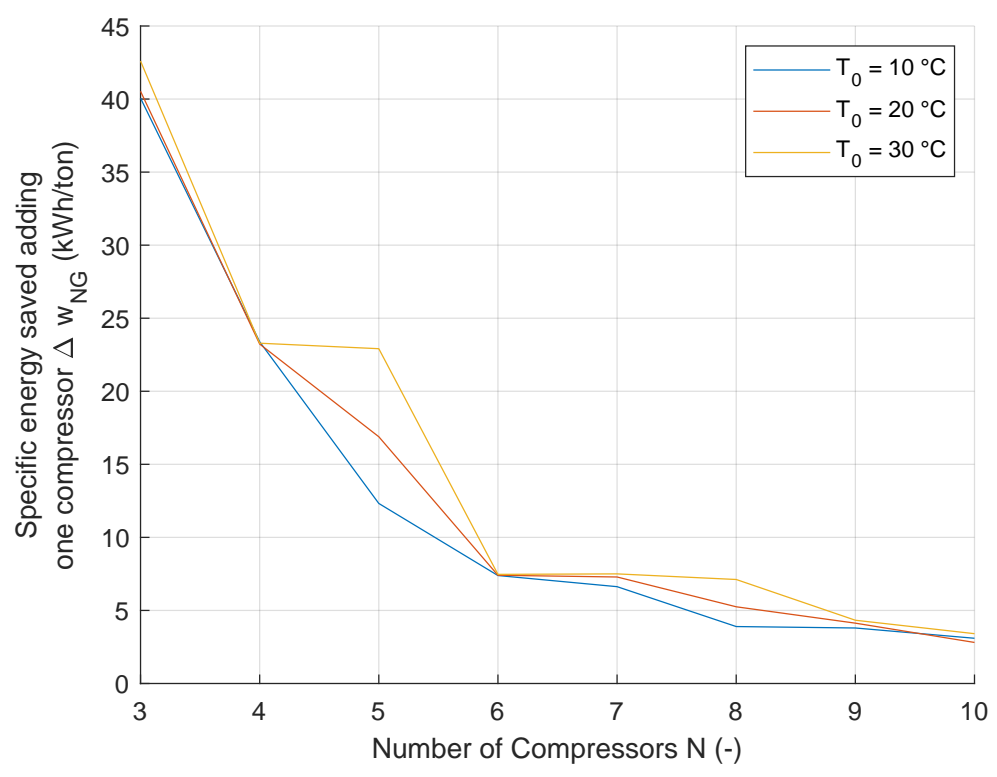

Figure 10. Energy savings gained by adding an extra compressor stage.

\section{Discussion}

The performance of the pure-component cascade process improves as stages are added. Commercial cascades generally use two or three pressure levels in each refrigerant circuit, six to nine stages in total. Figure 8 shows that if approximately 11 stages are used, the performance of the pure-component process equals the MFC process. The results displayed in Figure 9 for the $20^{\circ} \mathrm{C}$ cases show that in moving from a six-stage process to a nine-stage process, additional levels should first be added in the ethylene circuit (2-3-2) and then the methane circuit (2-3-3) before the propane (3-3-3).

The optimization parameters considered in this study are limited to temperatures, pressures and exchanger configurations. The design of individual equipment items is not considered, and no cost-based optimization is presented. However, the use of a fixed pressure drop per stream in the 
heat exchangers allows the optimization routine to eliminate inefficient stages of heat transfer. Table 2, for example, shows that in the optimized 3-4-4 process, only 15 heat exchangers are used out of a maximum possible number $\left(\sum j_{n}\right)$ of 21 . Although the level of pressure loss associated with each heat exchanger and the minimum temperature approach used have not been optimized against equipment costs in this study, they have been set to a conservative/achievable level resulting in a realistic trade-off between efficiency and complexity.

The design of the open intercoolers used in each refrigerant circuit is idealized in the models developed. Compared to the generally conservative assumptions discussed above, a more detailed analysis of the design of these items of equipment may show that additional pressure drop would be required to implement this design in practice, which would in turn add a small margin in the calculated optimum energy consumption presented here.

Optimizing this type of process is difficult in conventional flow sheet-based modeling programs such as HYSYS because of the time-consuming nature of the flow sheet modifications needed to adjust the flow sheet when items of equipment are added and removed. By contrast, the algorithm developed in MATLAB is able to consider a large number of potential flow sheet options based on a simple framework of design parameters. It is, therefore, a more reliable tool when global minimum energy consumption is required. The results presented in Figures $4-6$ show both the complexity of the optimization problem presented and the efficient way in which the hybrid algorithm developed for this study is able to find the global minimum power. The findings of the verification study conducted in HYSYS also show that the algorithm developed in MATLAB is consistent and reliable.

\section{Conclusions}

The aim of the study was to develop a method to systematically evaluate the process performance for a pure-component cascade LNG liquefaction process and find the optimal design for all relevant compression stages. The hybrid optimization algorithm used in this study has been shown to quickly and reliably find the global minimum power for the process. The approach is well suited to optimization problems where a large number of alternative process flow sheet alternatives exist and could be applied in other processes where energy efficiency is important.

Although the parameters used in this study represent realistic/achievable process equipment design, the trade-off between capital costs and process performance is not considered here. Therefore, the optimum configurations presented here may not represent the lowest overall lifecycle costs for a real process design.

Additional optimization parameters relating to equipment performance could be added to allow a cost-based optimization. This study considered only the optimization of energy efficiency of a process with fixed equipment design parameters.

This study shows that approximately 11 stages are required for a pure-component cascade scheme to equal the energy efficiency of an MFC process using three refrigerant mixtures. The optimum configuration of this scheme is found to be 3-4-4 as described in Table 2.

Acknowledgments: The publication charges for this article have been funded by a grant from the publication fund of UiTThe Arctic University of Norway.

Author Contributions: O.E. is responsible for the development of the optimization routine and is the main author of this article. E.B. is responsible for the initiation of this research work; supporting and guiding of the development of the optimization routine; and supporting the writing of this article. S.J. is responsible for the verification of the optimization results and supported the writing of this article.

Conflicts of Interest: The authors declare no conflict of interest. 


\section{References}

1. Lim, W.; Choi, K.; Moon, I. Current Status and Perspectives of Liquefied Natural Gas (LNG) Plant Design. Ind. Eng. Chem. Res. 2013, 52, 3065-3088.

2. Bauer, H.C. Mixed fluid cascade, experience and outlook. In Proceedings of the 8th Global Congress on 12AIChE-2012 AIChE Spring Meeting, Houston, TX, USA, 1-5 April 2012.

3. Pettersen, J.; Nilsen, Ø.; Vist, S.; Giljarhus, L.E.N.; Fredheim, A.O.; Aasekjær, K.; Neeraas, B.O. Technical and Operational Innovation for Onshore and Floating LNG. In Proceedings of the 17th International Conference \& Exhibition on Liquefied Natural Gas (LNG 17), Houston, TX, USA, 16-19 April 2013; pp. 1-12.

4. Kamalinejad, M.; Amidpour, M.; Naeynian, S.M.M. Optimal Synthesis of a Cascade Refrigeration System of LNG Through MINLP Model for Pure Refrigerant Cycles. Gas Process. J. 2014, 2, 51-68.

5. Barnés, F.J.; King, C.J. Synthesis of Cascade Refrigeration and Liquefaction Systems. Ind. Eng. Chem. Process Des. Dev. 1974, 13, 421-433.

6. Andress, D. The Phillips Optimized Cascade LNG Process: A Quarter-of-a-Century of Improvements; Phillips Petroleum Company: Bartlesville, OK, USA, 1996.

7. Wang, J.; Smith, R. Synthesis and Optimization of Low-Temperature Gas Separation Processes. Ind. Eng. Chem. Res. 2005, 44, 2856-2870.

8. Austbø, B.; Løvseth, S.W.; Gundersen, T. Annotated bibliography-Use of optimization in LNG process design and operation. Comput. Chem. Eng. 2014, 71, 391-414.

9. Bosma, P.; Nagelvoort, R.K. Liquefaction Technology; Developments through History. In Proceedings of the 1st Annual Gas Processing Symposium, Doha, Qatar, 10-12 January 2009; Volume 1, pp. 19-31.

10. Eaton, A.; Hernandez, R.; Risley, A.; Hunter, P.; Avidan, A.; Duty, J. Lowering LNG unit costs through large and efficient LNG liquefaction trains-What is the optimal train size. In Proceedings of the AIChE Spring Meeting, New Orleans, LA, USA, 25-29 April 2004; pp. 25-29.

11. Bell, I.H.; Wronski, J.; Quoilin, S.; Lemort, V. Pure and Pseudo-pure Fluid Thermophysical Property Evaluation and the Open-Source Thermophysical Property Library CoolProp. Ind. Eng. Chem. Res. 2014, 53, 2498-2508.

12. Jackson, S.; Eiksund, O.; Brodal, E. Impact of Ambient Temperature on LNG Liquefaction Process Performance: Energy Efficiency and $\mathrm{CO}_{2}$ Emissions in Cold Climates. Ind. Eng. Chem. Res. 2017, 56, 3388-3398.

(C) 2018 by the authors. Licensee MDPI, Basel, Switzerland. This article is an open access article distributed under the terms and conditions of the Creative Commons Attribution (CC BY) license (http://creativecommons.org/licenses/by/4.0/). 